\title{
Evaluating the Impacts of Climate Change on Diurnal Wind Power Cycles using Multiple Regional Climate Models
}

\author{
Scott D. Goddard, ${ }^{1}$ Marc G. Genton, ${ }^{2}$ Amanda S. Hering, ${ }^{3, *}$ and \\ Stephan R. Sain ${ }^{4}$
}

October 14, 2014

\begin{abstract}
Electrical utility system operators must plan resources so that electricity supply matches demand throughout the day. As the proportion of wind-generated electricity in the U.S. grows, changes in daily wind patterns have the potential either to disrupt the utility or increase the value of wind to the system over time. Wind power projects are designed to last many years, so at this timescale, climate change may become an influential factor on wind patterns. We examine the potential effects of climate change on the average diurnal power production cycles at 12 locations in North America by analyzing averaged and individual output from 9 high-resolution regional climate models (RCMs) comprising historical (19711999) and future (2041-2069) periods. A semi-parametric mixed model is fit using cubic B-splines, and model diagnostics are checked. Then, a likelihood ratio test is applied to test for differences between the time periods in the seasonal daily averaged cycles, and agreement among the individual RCMs is assessed. We investigate the significant changes by combining boxplots with a differencing approach and identify broad categories of changes in the amplitude, shape, and position of the average daily cycles. We then discuss the potential impact of these changes on wind power production.
\end{abstract}

Some keywords: Climate Change, Diurnal Power Cycles, Regional Climate Models, Wind Energy

Short title: Climate Change Impacts on Diurnal Wind Power Cycles

\footnotetext{
${ }^{1}$ Department of Statistics, Texas A\&M University, College Station, TX 77843

${ }^{2}$ Division of Computer, Electrical and Mathematical Sciences and Engineering, King Abdullah University of Science and Technology, Thuwal 23955, Saudi Arabia

${ }^{3}$ Department of Applied Mathematics and Statistics, Colorado School of Mines, Golden, CO 80401,

*Corresponding Author E-mail: ahering@mines.edu

${ }^{4}$ The Climate Corporation, San Francisco, CA 94103
} 


\section{Introduction}

Wind-generated electricity is a promising source of energy with increasingly widespread installation. As of June 2014, the United States' wind power capacity stood at 61,946 megawatts (MW) with over 46,300 wind turbines (AWEA 2014). But wind power's near-term growth may be more impressive still. The U.S. Department of Energy (DoE) has projected that $20 \%$ of the U.S. electricity demand could be met with wind by 2030 (DoE 2008).

Many current wind power projects and those under development should remain operational well beyond that time (Pryor and Barthelmie 2011). These projects will supply meteorologically derived energy throughout a period of ongoing climatic evolution, which has prompted a great deal of research. Pryor and Barthelmie (2010) reviewed much of the literature up until 2010, and at that time, they found very little evidence to suggest any detectable change in the wind resource. However, they conclude that more research is certainly warranted. Mixed results on the effects of climate change on wind speed patterns have also been reported in various regional studies (Pryor et al. 2005a; Pryor et al. 2005b; Pryor et al. 2005c; Pryor et al. 2012b; Najac et al. 2009; Nolan et al. 2012; Pryor and Barthelmie 2011; Pryor et al. 2009; Breslow and Sailor 2009; Segal et al. 2001; Sailor et al. 2008).

In this work, we seek to extend these prior results in three substantial ways. First, we use nine regional climate models (RCMs) from the North American Regional Climate Change Assessment Program (NARCCAP) (Mearns et al. 2011) to obtain greater specificity for the region and greater precision in the results across multiple models. Second, we analyze the impact of climate change on the diurnal cycle of wind, which will have a great impact on wind energy integration as the proportion of wind energy in utility portfolios grows in the future. Finally, we develop a novel statistical approach that accounts for the periodic nature of the daily functional cycles, and we adjust our results to control the false discovery rate, making 
them robust to erroneous rejections of the null hypothesis. With these extensions, we seek to investigate the following questions regarding future wind-power capabilities: (1) will average power generation diurnal cycles at twelve specific wind-energy locations change significantly due to climate change, and (2) what types and magnitudes of changes are expected?

In the next section, we review the current literature on climate change projections for the wind resource in terms of models used and quantities evaluated. We also review and discuss the literature on the ability of the NARCCAP RCMs to reproduce historical wind climates and diurnal wind patterns. In Section 3, we describe the RCM data and present the general semi-parametric mixed model and likelihood ratio test that can be used to test for changes in projected wind power cycles. Section 4 presents our results, and we conclude in Section 5 with a discussion of the results' implications and limitations.

\section{Literature Review}

In previous work, wind speed projections from global general circulation models have been statistically downscaled in order to obtain data with a sufficiently high-resolution for the research questions at hand (Pryor et al. 2005a; Pryor et al. 2005b; Najac et al. 2009; Sailor et al. 2008). But regional climate models nested within general circulation models can be run at even finer spatial resolutions, yielding sharper analyses of local effects. Of those studies that do use RCMs, most rely on data from one or two nestings (Nolan et al. 2012; Segal et al. 2001; Pryor et al. 2005c; Pryor et al. 2009; Pryor et al. 2012b). Comparing the observed wind climates with that produced by climate models is inherently difficult due to a lack of spatial and temporally dense reference wind observations, but recently, Pryor et al. (2012a) compared the NARCCAP RCM winds at 10 meters above ground level with the North American Regional Reanalysis (NARR) (Mesinger et al. 2006). They found that 
winds in all of the RCMs were positively biased relative to the NARR, but no one RCM could be considered "uniformly best." They suggest that in any analysis, a full suite of climate models should be considered, as we do here.

Previous analyses have also primarily examined climate change impacts on summary statistics of wind speed distributions (Pryor et al. 2005a; Pryor et al. 2005b; Pryor et al. 2005c; Najac et al. 2009; Nolan et al. 2012; Pryor et al. 2009; Breslow and Sailor 2002; Segal et al. 2001; Sailor et al. 2008); aggregate measures of "wind resource" (Pryor et al. 2005c; Clifton and Lundquist 2012); or the frequency of extreme wind events (Pryor et al. 2005c; Pryor et al. 2012b). While still important, these studies may overlook changes in wind behavior that can strongly impact wind power projects. For example, even if the mean wind speed is projected to remain constant, transformations of daily, periodic wind patterns may either reduce or increase the value of wind at a given location (Peterson et al. 1998). A favorable wind resource is one that is not only strong but that also aligns well with electricity demand, or load. Since no economically viable means of storing wind energy exists (Genton and Hering 2007), wind energy is distributed as it is generated. If high winds occur consistently during peak loads, grid operators can reduce their reliance on expensive "on demand" generators (Diakov and Short 2011). Alternatively, if high winds peak at night when the load is low, wind power may need to be curtailed, resulting in wasted generation opportunities. Thus, a horizontal shift in the diurnal power cycle does not change the mean wind power, but the value of the wind may increase if the cycle more closely matches the load.

Although Pryor et al. (2012a) found that the NARCCAP RCMs possess mixed skill in simulating historical wind climatology, our focus on significant differences between current and future diurnal power cycles, rather than on absolute quantities, results in the cancellation of any uniform model biases (such as a model that uniformly simulates winds that are too 
high or too low). Moreover, the performance of RCMs in reproducing historical climate is not necessarily an indicator that the future runs of RCMs lack skill in detecting changes in climate (Santer et al. 2009; Knutti et al. 2010). Notably, other work has shown a correlation between diurnal wind speed patterns and vertical temperature profile (He et al. 2013), as well as a consistency between the diurnal speeds and temperature fluctuations in RCM simulations (Zhang and Zheng 2004).

Thus, we define our primary variable of interest to be the diurnal wind power cycle. Many studies have documented the presence of diurnal cycles in wind (e.g., Brown et al. 1984; Zhang and Zheng 2004; Gneiting et al. 2006; Hering and Genton 2010; He et al. 2013). The relationship between wind speed and power production depends on the wind turbine model and is not a deterministic transformation (Pinson et al. 2008; Jeon and Taylor 2012; Lee et al. 2015a). However, given that we are interested in long-term average diurnal behaviors, we assume that this is a smooth relationship. Power generally increases with the cube of wind speed until it reaches the rated capacity of the turbine, as illustrated in the left panel of Figure 1. The power output then remains constant and shuts down when winds become high enough to damage the turbine.

A diurnal power production curve is obtained by plotting power against hour of the day. Here and throughout, time is represented on a 24 -hour scale with hour 25 corresponding to the following day's 1:00 AM. When daily variability in power production exhibits a cyclical pattern, multiple days' curves can be averaged into a central curve to represent the diurnal cycle of power production. The right panel of Figure 1 displays 29 daily power curves smoothed into a single diurnal power cycle and a typical average diurnal load cycle for the state of Texas (ERCOT 2013). Although the turbine output cycle and the load cycle are plotted on different scales, the graph illustrates that generated power is currently more valuable in late morning through the evening hours when demand is high. In the future, load 
cycles may differ due to changing electricity usage patterns, such as an increase in the number of electric vehicles plugged in overnight or a widespread redefinition of the weekly work schedule, but the current load data illustrates the importance of the relationship between wind power supply and load.

\section{Data and Methods}

\subsection{Data}

NARCCAP nests 6 regional climate models (RCMs) within 4 atmosphere-ocean general circulation models in a fractional factorial design to produce 12 catalogs of climate data (Mearns et al. 2011). Each of these 12 data catalogs is produced on roughly the same spatial domain (most of the land-area in North America and some of the surrounding ocean) with approximately $50 \times 50 \mathrm{~km}$ grid resolution. The time domain consists of the years 1971-2000 ("historical projections") and 2041-2070 ("future projections"), and the temporal resolution for the meridional and zonal wind components is a 3-hour interval. Atmospheric conditions for the historical set of years use historic green-house gas emissions, while the future runs of each RCM are based on the A2 emissions scenario (Nakicenovic and Swart 2000). Developed by the Intergovernmental Panel on Climate Change, this scenario depicts a relatively strong surface warming trend so that the climate change signal will be clear in future projections.

NARCCAP notation typesets a model's AOGCM in upper-case and its RCM in lowercase. The 9 data catalogs that we use are those that were published and complete at the time of this research. They are: the RCM3cgcm 3 model, the CRCMcgcm3 model, the HRM3hadcm3 model, the WRFGcgcm3 model, the WRFGccsm model, the MM5Iccsm model, the HRM3gfdl model, the CRCMccsm model, and the RCM3gfdl model. Exploratory data analysis revealed that three catalogs provide fewer than 60 years of data; we therefore 
trimmed all the catalogs' data to 58 years for consistency. Thus, the temporal domains of the data are 1971-1999 and 2041-2069.

These models report $u$ (east-west) and $v$ (north-south) wind components at 10 meters above ground level, which we convert to wind speed. Typical wind turbine hubs range in height from 30 to 120 meters, but common utility-grade turbines are 80 meters. Wind speed has therefore been transformed to an $80 \mathrm{~m}$ height using a standard logarithmic transformation with a roughness length of 0.01 , which is common in regions with wind projects (Archer and Jacobson 2003; Yoder et al. 2014). Pérez et al. (2005) showed that wind profiles may depend on daily and seasonal cycles, but Yoder et al. (2014) found very little effect of the type of transformation and roughness coefficient on forecasting wind power for a short-term horizon. Thus, this transformation should yield a reasonable depiction of $80 \mathrm{~m}$ wind speeds for comparing current and future projections.

Twelve locations in North America are selected on the basis of being important windenergy sites (currently, prospectively, or both) and being well distributed across the spatial domain. These sites are listed in Table 1 and are labeled in the upper-left panel of Figure 2. Since both existing and proposed wind developments may benefit from an analysis of the expected impact of climate change on their diurnal power cycles, eight of the 12 sites that we use already have large-scale wind-generation facilities. The other four (NL, BC, TN, and WY) are locations where wind is a promising and prospective resource for power. Some of the sites are high profile: Altamont, CA, was one of the pioneering sites in wind energy development, and the proposed off-shore project at Cape Cod, MA has received considerable media attention.

The $80 \mathrm{~m}$ wind speeds for the RCM grid boxes containing each of the 12 sites are converted into kilowatts of power using the power curves from one of three different turbines, depending on location: the Vesta V100 2.6 MW turbine, the Vesta V90 3.0 MW turbine, and the Siemens 
SWT 120 3.6 MW turbine. Power curves for these turbines are shown in the left panel of Figure 1. The 2.6 MW power curve transform is applied to the relatively low-wind sites of TN, CA, and NL because of its lower cut-in speed. Wind speeds for the AB, TX, WY, BA, IL, MN, ON, and OR locations are transformed using the 3.0 MW power curve, a turbine typically used in high-wind locations. The 3.6 MW turbine is designed for off-shore use, and its power curve is applied to the MA wind speed data.

\subsection{Models}

The analyses are performed using yearly and ensemble-averaged seasonal data. More explicitly, we consider the data at location $h(h$ in $\{1, \ldots, 12\})$ in catalog $i$ ( $i$ in $\{1, \ldots, 9\})$. In year $j(j$ in $\{1, \ldots, 58\})$, the $l$ th-hour $(l$ in $\{1, \ldots, 8\})$ wind speed is averaged over all days in season $k(k$ in $\{1, \ldots, 4\})$ to give a seasonal year- $j, l$ th-hour mean speed. In this way, twenty-nine curves for each of the historical and future data are formed. The vertical transformation and then the power transformation are applied, producing 58 diurnal power curves for each season, location, and catalog. The averaged-catalog data is produced by pooling and averaging the year- $j$, season- $k$ mean diurnal curves from the 9 catalogs. Each power curve is constrained to begin and end at the same point by appending the day's first value to the end of the curve, such that each of the final curves contain nine points.

To check for changes in diurnal power curves, we perform a hypothesis test on a subset of regression coefficients in a linear semi-parametric model. Not only does building a model provide a natural means for performing the hypothesis test, but it also provides the added advantages of making test assumptions simple to check and supplying parameter estimates for possible additional insight. The linear model is fit to the curves using cubic B-spline basis functions (Piegl and Tiller 1995). B-splines are both flexible and parsimonious, and testing their coefficients is straightforward. For these reasons, we prefer B-splines for this 
analysis over fitting Fourier series, as is commonly done with atmospheric variables (Collier and Bowman 2004). We consider the 58 mean curves for location $h$ and season $k$. A linear model is created for the 29 historical curves (indicated with a subscript 1) using the form

$$
\mathbf{y}_{1}=\mathbf{X}_{S} \mathbf{b}_{1}+\mathbf{Z}_{1} \mathbf{u}_{1}+\mathbf{e}_{1}
$$

Here $\mathbf{y}_{1}=\left(y_{1 j l}\right)$ is the vector of all curves for the historical data sample, where $y_{1 j l}$ refers to the power from the $l$ th time point in the $j$ th year's curve $(j=1, \ldots, 29, l=1, \ldots, 9) . \mathbf{X}_{S}$ is a matrix constructed using the basis functions of cubic B-splines (hence the subscript $S$ ) with $d$ degrees of freedom. $\mathbf{X}_{S}$ is a block matrix formed by stacking the $9 \times d$ matrix $\left(X_{l m}\right) 29$ times over, where $X_{l m}$ refers to the $m$ th element in the $l$ th basis function (for $m=1, \ldots, d$ ). The vector of coefficients is $\mathbf{b}_{1}=\left(b_{1 m}\right)$, where $b_{1 m}$ refers to the $m$ th spline coefficient. $\mathbf{Z}_{1}$ is a design matrix that provides for a yearly random effect, constructed as a diagonal block matrix of the vector $\mathbf{1}_{9}$ (a vector of nine 1 's). The $\mathbf{u}_{1}=\left(u_{1 j}\right)$ is a vector of random effects where $u_{1 j}$ is the random effect in year $j$, and $\mathbf{e}_{1}=\left(e_{1 j l}\right)$ is a vector of random errors, where $e_{1 j l}$ refers to the random error in the $l$ th time point in the $j$ th year's curve. For each season and location, $d$ is set at the value in $\{4, \ldots, 9\}$ that maximizes the normality of the residuals. The entries of $\mathbf{X}_{S}$ are generated by the "bs" function in the "splines" package in R. This package implements cubic spline recursion formulas found in Chambers and Hastie (1992).

The random effect term, $\mathbf{Z}_{1} \mathbf{u}_{1}$, is added to the model both for contextual and statistical reasons; we might well expect the climate models to allow some years to be "windier" than others or to evidence a "year effect." $\mathbf{Z}_{1}$ allows each annual mean curve to randomly shift in a uniform manner, where the random vector, $\mathbf{u}_{1}$, represents 29 independent draws from a normal distribution with mean 0 and variance $\sigma_{u}^{2}$. Thus, by modeling yearly effects, the term $\mathbf{Z}_{1} \mathbf{u}_{1}$ reduces the correlation between errors within the same year. We then assume that the 
$\mathbf{e}_{1}$ values are independent draws from a normal distribution with mean 0 and variance $\sigma^{2}$. The right panel of Figure 1 illustrates an example of $\mathbf{y}_{1}$, the twenty-nine dotted, gray curves, and $\mathbf{X}_{S} \hat{\mathbf{b}}_{1}$, the solid curve.

The quantities $\mathbf{y}_{2}, \mathbf{b}_{2}, \mathbf{e}_{2}, \mathbf{Z}_{2}$, and $\mathbf{u}_{2}$ are defined analogously for the future data sample. Equation (1) is extended to include both the 29 historical and the 29 future mean curves with the model:

$$
\mathbf{y}_{2}=\mathbf{X}_{S} \mathbf{b}_{1}+\mathbf{X}_{S} \mathbf{b}_{2}+\mathbf{Z}_{2} \mathbf{u}_{2}+\mathbf{e}_{2}
$$

In this form, it is clear that the mean structure for the historical data sample is $\mathbf{X}_{S} \mathbf{b}_{1}$, and it is $\mathbf{X}_{S} \mathbf{b}_{1}+\mathbf{X}_{S} \mathbf{b}_{2}$ for the future data sample. If $\mathbf{b}_{2}=\mathbf{0}$, then there is no difference between the means of the historical and future populations; if $\mathbf{b}_{2} \neq \mathbf{0}$, then an adjustment is needed to fit future curves beyond what is used to fit the historical curves.

\subsection{Tests}

A likelihood ratio test is used to test the null hypothesis that $\mathbf{b}_{2}=\mathbf{0}$. The test statistic, $W$, is the ratio of the likelihood function of the data maximized over the constrained parameter space $\left(\mathbf{b}_{2}=\mathbf{0}\right)$ to the likelihood maximized over an unconstrained parameter space. The maximization is done numerically. Under the null hypothesis and certain assumptions on the errors (described below), $-2 \log W$ is approximately $\chi_{d}^{2}$ distributed, where $d$ is the degrees of freedom of the basis functions in $\mathbf{X}_{S}$.

A separate model is fit, and the test is performed at each of the 12 locations for each of four seasons, resulting in 48 tests. To control the false discovery rate, we use a method described by Benjamini and Yekutieli (2001) that accounts for the dependency among tests. First, we let $q$ be the desired false discovery rate. The $p$-values of the 48 tests are ordered 
as $p_{(1)} \leq p_{(2)} \leq \cdots \leq p_{(48)}$. Then, letting

$$
k=\max \left\{i: p_{(i)} \leq \frac{i q}{48 \sum_{j=1}^{48} \frac{1}{j}}\right\}
$$

we reject all null hypotheses corresponding to $p$-values that are less than or equal to $p_{(k)}$, i.e., $\left\{p_{(1)}, p_{(2)}, \ldots, p_{(k-1)}, p_{(k)}\right\}$. This controls the expected value of the false discovery rate to be less than or equal to $q$.

The likelihood functions used to compute the test statistic, $W$, are derived under the assumption that the errors have a normal $\left(\mathbf{0}, \sigma^{2} \mathbf{I}\right)$ distribution and that the random effects are independent of the errors and have a normal $\left(\mathbf{0}, \sigma_{u}^{2} \mathbf{I}\right)$ distribution. The residuals of this model are defined as

$$
\hat{\mathbf{e}}=\left[\begin{array}{c}
\mathbf{y}_{1} \\
\mathbf{y}_{2}
\end{array}\right]-\left[\begin{array}{cc}
\mathbf{X}_{S} & \mathbf{0} \\
\mathbf{X}_{S} & \mathbf{X}_{S}
\end{array}\right]\left[\begin{array}{c}
\hat{\mathbf{b}}_{1} \\
\hat{\mathbf{b}}_{2}
\end{array}\right]-\left[\begin{array}{cc}
\mathbf{Z}_{1} & \mathbf{0} \\
\mathbf{0} & \mathbf{Z}_{2}
\end{array}\right]\left[\begin{array}{c}
\hat{\mathbf{u}}_{1} \\
\hat{\mathbf{u}}_{2}
\end{array}\right] .
$$

The assumption of normality is checked on $\hat{\mathbf{e}}$ and $\hat{\mathbf{u}}=\left[\begin{array}{cc}\hat{\mathbf{u}}_{1}^{T} & \hat{\mathbf{u}}_{2}^{T}\end{array}\right]^{T}$ using the Shapiro-Wilk test (Shapiro and Wilk 1965). Those residuals whose $p$-values are greater than 0.05 or 0.01 exhibit "strong" or "moderate" adherence to normality, respectively. Homoscedasticity and independence for $\hat{\mathbf{e}}$ are checked visually with boxplots. The residuals are first classified by hour-of-day for separate boxes in the plot and then by year-in-sample. Homoscedasticity for $\hat{\mathbf{u}}$ is checked using a dot plot that uses the year-in-sample as an index. Independence is validated using the Wald-Wolfowitz runs test (Wald and Wolfowitz 1940).

The parameters $\sigma_{u}^{2}$ and $\sigma^{2}$ for each season-location model are the variance of the annual random effects and errors, respectively. An estimate of $\hat{\sigma}^{2}$ that greatly exceeds $\hat{\sigma}_{u}^{2}$ would indicate that random noise dominates the yearly random effect in the diurnal curves, making 
a random effect term less important. In addition, if each season-location's fitted model produces estimates of $\sigma_{u}^{2}$ and $\sigma^{2}$ that are approximately equal to those of all other seasonlocations, then this similarity might argue in favor of a model that assumes each seasonlocation's errors and random effects are drawn from a common distribution. However, by fitting Equation (1) to to each combination of season and location individually, we allow not only the diurnal cycles to differ but also the variability in the second-order behavior of random effects and random noise to change. With wind data, this is generally warranted since an increase in the mean wind tends to correspond with an increase in variability. Both $\hat{\sigma}_{u}^{2}$ and $\hat{\sigma}^{2}$ are estimated using maximum likelihood estimation with a constraint to ensure non-negative estimates.

\section{Results}

The hypothesis test described above tests for a significant change in the diurnal power cycles between the historical and future samples, each of which is averaged over 9 regional climate models. Figure 2 displays the results of the tests for each season. Each location's plotted symbol depicts a disc surrounded by a segmented ring. The disc is shaded proportionally to the smallest false discovery rate (an increased likelihood of detecting significance simply by

random chance), $q$, at which the test based on the averaged RCMs is significant. The values of $q$ given in the legend correspond to the $q$ in Equation (2), and even when controlling for multiple testing, $77 \%$ of the tests are statistically significant at $q=0.05$. The results are most dramatic for the summer season (11/12 significant), followed by fall, spring, and winter with 10, 9, and 7 significant results, respectively. Only TX and NL show significant differences in every season, but these differences are not consistent across seasons. For example, in NL (summer), the power cycle is expected to shift upward with respect to the historical climate, 
but in spring, the amplitude of the average power cycle increases.

The outer ring of each symbol is divided into 9 segments corresponding to the RCMs, where their order follows that of the RCM names listed in Section 3.1, starting at the "1 o'clock" position and continuing clockwise. Just like the inner disc, each segment is shaded proportionally to its level of significance when the model is fit and hypothesis test performed using only the data from one RCM. The purpose of this is to indicate the diversity of test results produced by the various RCMs and illustrate the similarity of the averaged-catalog test result to those of the individual models. The results demonstrate that consensus among all of the individual RCMs is rare. In some cases, averaged-catalog tests that are significant at moderate values of $q$, like 0.05 , appear to reflect a compromise between strongly significant RCM tests and wholly insignificant ones, as with ON (autumn) and TX (spring). It can also be seen that significant test results in different seasons and locations are frequently influenced by the same small number of RCMs. Take, for example, the region at the "9 o'clock" position of the ring, which is significant at $q=0.001$ for every single test. This region corresponds to the "HRM3gfdl" RCM, which largely outputs wind speeds lower than those of the other RCMs. However, this one significant test result does not overcome all of the others as evidenced by the non-significant test results of the averaged catalogs for MN (winter), IL (winter), WY (spring), etc.

Figure 3 displays a sample of plots that are used to check the likelihood ratio test assumptions for the averaged-catalog tests at a specific season-location: MA (summer). The upper two plots show normal probability plots for all values of $\hat{\mathbf{u}}$ (left) and $\hat{\mathbf{e}}$ (right). In both plots, the assumption of normality is reasonably justified. The bottom-left plot displays boxplots for the values of $\mathbf{e}$ across the diurnal curve wherein it is evident that the variances of the errors are reasonably alike at each time of day. The full model diagnostics described in Section 3 were performed for each model's residuals from the averaged-catalog fit, resulting 
in our finding that 7 models strongly adhere to all the assumptions and 7 of them moderately adhere, while the remaining 34 contain some discrepancies to at least one assumption. The bottom-right plot shows the distributions of the values of $\hat{\sigma}_{u}$ and $\hat{\sigma}$ from all 48 fitted models. The variability in these distributions indicates that the variance components $\sigma_{u}^{2}$ and $\sigma^{2}$ are distinct for the different seasons and locations, as anticipated. Also, although the distribution of $\hat{\sigma}_{u}$ is concentrated at lower values than that of $\hat{\sigma}$, it is evident from the distribution's positive values that including the yearly random effect term in the models is generally warranted. A possible example of an uncommonly strong yearly random effect is shown by the low, outlying points in the plot of ON (spring) in Figure 4.

Although the hypothesis test we use is robust to moderate departures from its underlying assumptions in the likelihood ratio test (particularly normality), we focus the remainder of our presentation on the 14 averaged-catalog season-locations that display either strong or moderate adherence to all of the assumptions, each of which also happens to be significant at $q=0.001$. These illustrate the typical differences observed between historical and future cycles. To examine these differences, a 70-year-differenced curve is created by subtracting the mean curve in 1971 (pointwise) from the mean curve in 2041. The same is done for the remaining 28 pairs of curves, creating a sample of 29 differenced curves. A boxplot of the differences for each hour of the day is plotted in Figure 4, along with the historical and future fitted B-spline diurnal cycles, shifted such that they overlay the boxplots. Note that when the historical fitted B-spline is above the future fitted B-spline, then the differenced boxplots tend to be below zero, and conversely, they tend to be above zero when the future curve is projected to increase over the historical climate. It is clear that for the season-location tests in Figure 4, the boxplots vary both in center and spread over the course of the day.

Table 2 classifies the observed changes in Figure 4 into three broad groups and reports the mean difference between the fitted splines and its standard error. We note that the mean 
differences summarize the average change in power across the entire diurnal cycle for one turbine. When scaled to a wind farm with tens or hundreds of turbines and accumulated over time, even small changes at the turbine level can equate to large losses or gains in power. For example, if ON has $3 \mathrm{MW}$ turbines installed, then 304 turbines are required to reach its installation level of $912 \mathrm{MW}$. This would equate to an average spring increase of $304 \times 7=2,128 \mathrm{MW}$ and an average decrease of $304 \times 40=12,160 \mathrm{MW}$ in the summer. It should be noted that these figures are calculated by using the power functions of modern turbines, which may not reflect any improvements in the power conversion efficiency of new or upgraded turbines in the future (Lee et al. 2015b).

The first two rows of Figure 4 correspond to Group 1, those season-location sites that demonstrate a nearly uniform shift in the projection curves, leading to higher or lower overall electricity production. WY in winter loses the greatest average number of watts, although the summer loss in MA may have a greater impact on the utility because electric loads are higher in the summer. The third and fourth rows of curves correspond to Group 2, those season-location sites whose projected cycles change in amplitude with higher peaks and lower troughs as in TX (autumn) or with lower peaks and higher troughs as in MN (autumn, spring). The expected change in the NL (spring) cycle is one of the best-case scenarios; not only does the amplitude of the projected cycle increase, but its peak occurs during the midday hours, which coincides with high loads.

The last row of Figure 4 displays more complex cases. In TX (spring), wind power cycles are expected to increase, which would generally be considered favorable, but the increases are more pronounced late at night when demand is low. Thus, curtailment of power developments may be necessary if more power is produced than can be distributed. The ON (summer) curve generally shows a downward shift, but the shift is not as great in the evening hours as it is the rest of the day. Finally, the average daily peak in power produced in ON (autumn) is 
projected to shift from the afternoon to the evening, but combined with an overall downward shift, this change is not likely to disrupt utility scheduling.

\section{Discussion}

To achieve the DoE estimates of meeting the $20 \%$ renewable goal by 2030, "US wind power capacity would have to reach more than 300 gigawatts (GW)" (DoE 2008). Consequently, wind energy capacity in the US would need to increase fivefold over a period of 16 years. Even though the DoE's estimates do not represent binding legislation, they demonstrate the nation's growing interest in wind power development. Such projects may operate well into the time frame of the NARCCAP projections. Countries like Denmark, Spain, and the U.K. already meet $30 \%, 18.1 \%$, and $11.3 \%$ of their energy needs with wind power, respectively, and at such penetrations, the diurnal cycle of wind becomes very important. Thus, projected changes in wind power could have strong implications in the near term for siting new wind power developments (Chou and Corotis 1981), maintaining existing developments, planning new electrical infrastructure, and investing in other renewable technologies. Furthermore, a projected adjustment in a location's diurnal cycle could have important implications for the creation of short-term statistical wind forecast models (Hering and Genton 2010; Zhu and Genton 2012; Pinson 2013), which are necessary for utility system integration.

Our rigorous testing framework could be applied to any site of interest and could easily be integrated into wind resource assessment studies. Instead of counting the number of RCMs whose output is consistent with a particular hypothesis to assess the likelihood of a climate change, as in Pryor et al. (2012a), we have developed a statistical hypothesis test that combines information from all of the RCMs, incorporates the periodic nature of daily power curves, and controls for the Type I error when testing many sites and locations. The 
wide diversity of test results illustrated by the hypothesis test results on the individual RCMs and the consistent bias of certain RCMs toward significant or insignificant results supports the rationale to use a central summary of the whole ensemble of RCMs to produce more balanced results. Although we have taken a simple average of their diurnal curves, it may be reasonable to develop a weighting system in future analysis that weights each RCM based on each one's ability to replicate wind climatology. Alternatively, the diversity in individual RCM test results may indicate the need to use a more robust central summary, such as a median diurnal curve, to allay the influence of possibly outlying curves.

This analysis may also be extended by accounting for the distribution of wind speed at each hour. Doing so would provide a distribution on diurnal cycles, rather than just the mean diurnal cycles employed here. In addition, performing the analysis with updated models that have higher temporal or spatial resolution or a larger suite of RCMs, such as CMIP5 (Taylor et al. 2012), may yield even better results. Adapting this method to evaluate projected changes in diurnal cycles for an entire region and for different proportions of wind-generated electricity would also be informative for long-range utility planning (Marquis et al. 2011).

In closing, we have demonstrated that different sites may expect different types of climate change impacts on their diurnal power cycles. These changes must be evaluated in conjunction with the electrical load. When increases in wind power are projected to correspond with high electric loads, the value of the wind increases. Alternatively, if power cycles are not projected to align well with loads, utilities may need strategies for handling excess electricity production, especially as the penetration of wind in a utility increases. A thorough comparison of power production cycles to load cycles assumes a knowledge of other important factors such as how electrical load cycles will change in the future, how turbine technology will improve, and how many new turbines will be installed. These areas need further study, and we make no attempt to calculate the actual value of changes to power 
production cycles. However, the present analysis establishes that such changes are likely to occur, and these time-dependent adjustments will affect power producers and consumers in a more complex way than what has previously been shown.

\section{Acknowledgements}

The authors wish to thank Tim Hoar and Ying Sun for programming support. This research was initiated in connection with the SIParCS program at the National Center for Atmospheric Science, which is managed by the University Corporation for Atmospheric Research under the sponsorship of the National Science Foundation. We also wish to thank the North American Regional Climate Change Assessment Program (NARCCAP) for providing the data used in this paper. NARCCAP is funded by the National Science Foundation (NSF), the U.S. Department of Energy (DoE), the National Oceanic and Atmospheric Administration (NOAA), and the U.S. Environmental Protection Agency Office of Research and Development (EPA). Finally, we would like to thank two anonymous reviewers for valuable comments and suggestions that have improved this article's content and presentation. 


\section{References}

American Wind Energy Association (AWEA). US Wind Industry Second Quarter 2014 Market Report (2014). Data downloaded 2014-10-3. Available at http://awea.files.cmsplus.com/FileDownloads/pdfs/2Q2014\%20AWEA\%20Market\%20Report\%20Public\%20V ersion $\% 20 . p d f$.

Archer C, Jacobson M. 2003. Spatial and temporal distributions of US winds and wind power at $80 \mathrm{~m}$ derived from measurements. Journal Geophysical Research: Atmospheres 108(D9): 1-20. DOI: 10.1029/2002JD002076

Benjamini Y, Yekutieli D. 2001. The control of the false discovery rate in multiple testing under dependency. The Annals of Statistics 29(4): 1165-1188.

Breslow P, Sailor D. 2002. Vulnerability of wind power resources to climate change in the continental United States. Renewable Energy 27(4): 585-598. DOI: 10.1016/S09601481(01)00110-0

Brown B, Katz R, Murphy A. 1984. Time series models to simulate and forecast wind speed and wind power. Journal of Applied Meteorology and Climatology 23(8): 1184-1195.

Chambers J, Hastie, T. 1992. Statistical Models in S. CRC Press: Boca Raton, FL.

Chou K, Corotis R. 1981. Simulation of hourly wind speed and array wind power. Solar Energy 26(3): 199-212. DOI: 10.1016/0038-092X(81)90204-8

Clifton A, Lundquist J. 2012. Data clustering reveals climate impacts on local wind phenomena. Journal of Applied Meteorology and Climatology 51(8): 1547-1557.

Collier J, Bowman K. 2004. Diurnal cycle of tropical precipitation in a general circulation model. Journal of Geophysical Research: Atmospheres 109(D17): 105-124. DOI: 


\subsection{9/2004JD004818}

Diakov V, Short W. 2011. The value of geographic diversity of wind and solar: A vector analysis. ASME Conference Proceedings 2011 1093-1100. DOI: 10.1115/IMECE201163880

Electric Reliability Council of Texas. http://www.ercot.com/gridinfo/load/load_hist [accessed 6 May 2013]

Genton M G, Hering A S. 2007. Blowing in the wind. Significance 4(1): 11-14.

Gneiting T, Larson K, Westrick K, Genton M G, Aldrich E. 2006. Calibrated probabilistic forecasting at the stateline wind energy center. Journal of the American Statistical Association 101(475): 968-979. DOI: 10.1198/016214506000000456

He Y, Monahan A H, McFarlane N A. 2013. Diurnal variations of land surface wind speed probability distributions under clear-sky and low-cloud conditions. Geophysical Research Letters 40(12): 1-7. DOI: 10.1002/grl.50575

Hering A S, Genton M G. 2010. Powering up with space-time wind forecasting. Journal of the American Statistical Association 105(489): 92-104 (2010). DOI: 10.1198/jasa.2009.ap08117

Jeon J, Taylor J W. 2012. Using conditional kernel density estimation for wind power density forecasting. Journal of the American Statistical Association, 107(497): 66-79. DOI: $10.1080 / 01621459.2011 .643745$

Knutti R, Furrer R, Tebaldi C, Cermak J, Meehl G. 2010. A. Challenges in combining projections from multiple climate models. Journal of Climate 23(10): 2739-2758.

Lee G, Ding Y, Genton M G, Xie L. 2015a. Power curve estimation with multivariate environmental factors for inland and offshore wind farms. Journal of the American Statistical Association in press. 
Lee G, Ding Y, Xie L, Genton M G. 2015b. A kernel plus method for quantifying wind turbine performance upgrades. Wind Energy in press.

Marquis M, Wilczak J, Ahlstrom M, Sharp J, Stern A, Smith J C, Calvert S. 2011. Forecasting the wind to reach significant penetration levels of wind energy. Bulletin of the American Meteorological Society 92(9): 1159-1171.

Mearns L. et al. The North American regional climate change assessment program dataset. http://www.narccap.ucar.edu/data/ [accessed 27 December 2012]

Mesinger F. et al. 2006. North American regional reanalysis. Bulletin of the American Meteorological Society, 87(3): 343-360.

Najac J, Boé J, Terray L. 2009. A multi-model ensemble approach for assessment of climate change impact on surface winds in France. Climate Dynamics 32(5): 615-634.

Nakicenovic N, Swart R. eds. 2000. Emissions Scenarios. Cambridge University Press: Cambridge, UK.

National Renewable Energy Laboratory. 20\% Wind Energy by 2030: Increasing Wind Energy's Contribution to US Electricity Supply. US Department of Energy: Washington DC.

Nolan P, Lynch P, McGrath R, Semmler T, Wang S. 2012. Simulating climate change and its effects on the wind energy resource of Ireland. Wind Energy 15(4): 593-608. DOI: $10.1002 /$ we.489

Peterson E L, Mortensen N G, Landberg L, Højstrup J, Frank H P. 1998. Wind power meteorology. Part I: Climate and turbulence. Wind Energy 1(S1): 25-45.

Pérez I, García M, Sánchez M, de Torre B. 2005. Analysis and parameterisation of wind profiles in the low atmosphere. Solar Energy 78(6): 809-821. DOI: 10.1016/j.solener.2004.08.024 
Piegl L, Tiller W. 1995. B-spline basis functions. The NURBS Book, 47-79. Springer: Berlin.

Pierrot M. The wind power: Wind turbines and wind farms database. http://www.thewindpower.net/windfarms_list_en.php [accessed 17 January 2013]

Pinson P. 2013. Wind energy: forecasting challenges for its operational management. Statistical Science, 28(4): 564-585.

Pinson P, Nielsen H Aa, Madsen H, Nielsen T S. 2008. Local linear regression with adaptive orthogonal fitting for the wind power application. Statistics and Computing, 18(1): $59-71$.

Pryor S, Barthelmie R. 2010. Climate change impacts on wind energy: A review. Renewable and Sustainable Energy Reviews 14(1): 430-437. DOI: 10.1016/j.rser.2009.07.028

Pryor S, Barthelmie R. Assessing climate change impacts on the near-term stability of the wind energy resource over the United States. Proceedings of the National Academy of Sciences of the United States of America 108(20): 8167-8171. DOI: 10.1073/pnas.1019388108

Pryor S, Barthelmie R, Schoof J. 2012a. Past and future wind climates over the contiguous USA based on the North American Regional Climate Change Assessment Program model suite. Journal of Geophysical Research: Atmospheres 117(D19) 119-135. DOI: 10.1029/2012JD017449

Pryor S, Schoof J, Barthelmie R. 2005a. Climate change impacts on wind speeds and wind energy density in northern Europe: Empirical downscaling of multiple AOGCMs. Climate Research 29: 183-198.

Pryor S, Schoof J, Barthelmie R. 2005b. Empirical downscaling of wind speed probability distributions. Journal of Geophysical Research: Atmospheres 110(D19): 109-120. DOI: 


\subsection{9/2005JD005899}

Pryor S, Barthelmie R J, Clausen N E, Drews M, MacKellar N, Kjellström E. 2012b. Analyses of possible changes in intense and extreme wind speeds over northern Europe under climate change scenarios. Climate Dynamics 38(1-2): 189-208.

Pryor S, Barthelmie R, Kjellström E. 2005c. Potential climate change impact on wind energy resources in northern Europe: Analyses using a regional climate model. Climate Dynamics 25(7-8), 815-835.

Pryor S C, Barthelmie R J, Young D T, Takle E S, Arritt R W, Flory D, Gutowski Jr W J, Nunes A, Roads J. 2009. Wind speed trends over the contiguous United States. Journal of Geophysical Research: Atmospheres 114(D14): 105-122. DOI: 10.1029/2008JD011416

R Core Team. R: A language and environment for statistical computing. ISBN 3-90005107-0. http://www.R-project.org/

Sailor D, Smith M, Hart M. 2008. Climate change implications for wind power resources in the Northwest United States. Renewable Energy 33(11): 2393-2406.

DOI: 10.1016/j.renene.2008.01.007

Santer B D, et al. 2009. Incorporating model quality information in climate change detection and attribution studies. Proceedings of the National Academies of Sciences of the United States of America 106(35): 778-783.

Segal M, Pan Z, Arritt R, Takle E. 2001. On the potential change in wind power over the US due to increases of atmospheric greenhouse gases. Renewable Energy 24(2): 235-243. DOI: 10.1016/S0960-1481(00)00194-4

Shapiro S, Wilk M. 1965. An analysis of variance test for normality (complete samples). 
Biometrika 52(3/4): 591-611.

Taylor K E, Stouffer R J, Meehl G A. 2012. An overview of CMIP5 and the experiment design. Bulletin of the American Meteorological Society 93(4): 485-498.

Wald A, Wolfowitz J. 1940. On a test whether two samples are from the same population. The Annals of Mathematical Statistics 11(2): 147-162.

Yoder M, Hering A S, Navidi W C, Larson K. 2014. Short-term forecasting of categorical changes in wind power with Markov chain models. Wind Energy, 17(9): 1425-1439.

Zhang D, Zheng W. 2004. Diurnal cycles of surface winds and temperatures as simulated by five boundary layer parameterizations. Journal of Applied Meteorology and Climatology 43(1): 157-169.

Zhu X, Genton M G. 2012. Short-term wind speed forecasting for power system operations. International Statistical Review 80(1): 2-23. 
Table 1: Names of the 12 selected sites, as well as the current status and approximate nominal capacity of the project(s) in megawatts based on Pierrot (2012).

\begin{tabular}{rrrrrr} 
& & \multicolumn{3}{c}{ Approximate capacity (MW) } \\
\cline { 3 - 6 } Abbreviation & Location name & Planned & Construction & Operational & Total \\
\hline BC & La Rumorosa, Baja, Mexico & - & - & 10 & 10 \\
TN & Oak Ridge, Tennessee & - & - & 29 & 29 \\
WY & Rawlins, Wyoming & - & - & 50 & 50 \\
NL & Monterrey, Nuevo Leon, Mexico & - & 161 & - & 161 \\
TX & McCamey, Texas & - & - & 565 & 565 \\
AB & Pincher Creek, Alberta, Canada & - & - & 691 & 691 \\
$\mathrm{CA}$ & Altamont, California & - & 201 & 620 & 821 \\
$\mathrm{ON}$ & Wolfe Island, Ontario, Canada & 714 & - & 198 & 912 \\
$\mathrm{MN}$ & Lake Benton, Minnesota & - & 200 & 1268 & 1468 \\
$\mathrm{IL}$ & Mendota, Illinois & - & 105 & 1595 & 1700 \\
$\mathrm{MA}$ & Cape Cod, Massachusetts & 2208 & - & 3 & 2211 \\
$\mathrm{OR}$ & Wasco, Oregon & - & 845 & 1716 & 2561 \\
\hline
\end{tabular}


Table 2: Classification of the season-location tests from Figure 4 into groups that characterize the type of change observed in the mean diurnal cycles. The mean difference between the future and historical diurnal cycles is given with its estimated standard error.

\begin{tabular}{|c|c|c|c|c|}
\hline Group & Change & Season-location & Direction & Mean difference (SE) \\
\hline \multirow[t]{5}{*}{1} & Vertical shift & $\mathrm{AB}$ (winter) & Downward & $-46(27)$ \\
\hline & & WY (autumn) & Downward & $-37(25)$ \\
\hline & & WY (winter) & Downward & $-136(43)$ \\
\hline & & NL (summer) & Upward & $54(9)$ \\
\hline & & MA (summer) & Downward & $-100(17)$ \\
\hline \multirow[t]{6}{*}{2} & Amplitude change & TX (autumn) & Increase & $10(10)$ \\
\hline & & NL (spring) & Increase & $33(6)$ \\
\hline & & MN (autumn) & Decrease & $-12(15)$ \\
\hline & & MN (spring) & Decrease & $-1(20)$ \\
\hline & & IL (summer) & Decrease & $0(8)$ \\
\hline & & ON (spring) & Decrease & $7(14)$ \\
\hline \multirow[t]{3}{*}{3} & Other changes & TX (spring) & Partial upward shift & $66(13)$ \\
\hline & & ON (summer) & Partial downward shift & $-40(5)$ \\
\hline & & ON (autumn) & Rightward/downward shift & $-39(12)$ \\
\hline
\end{tabular}



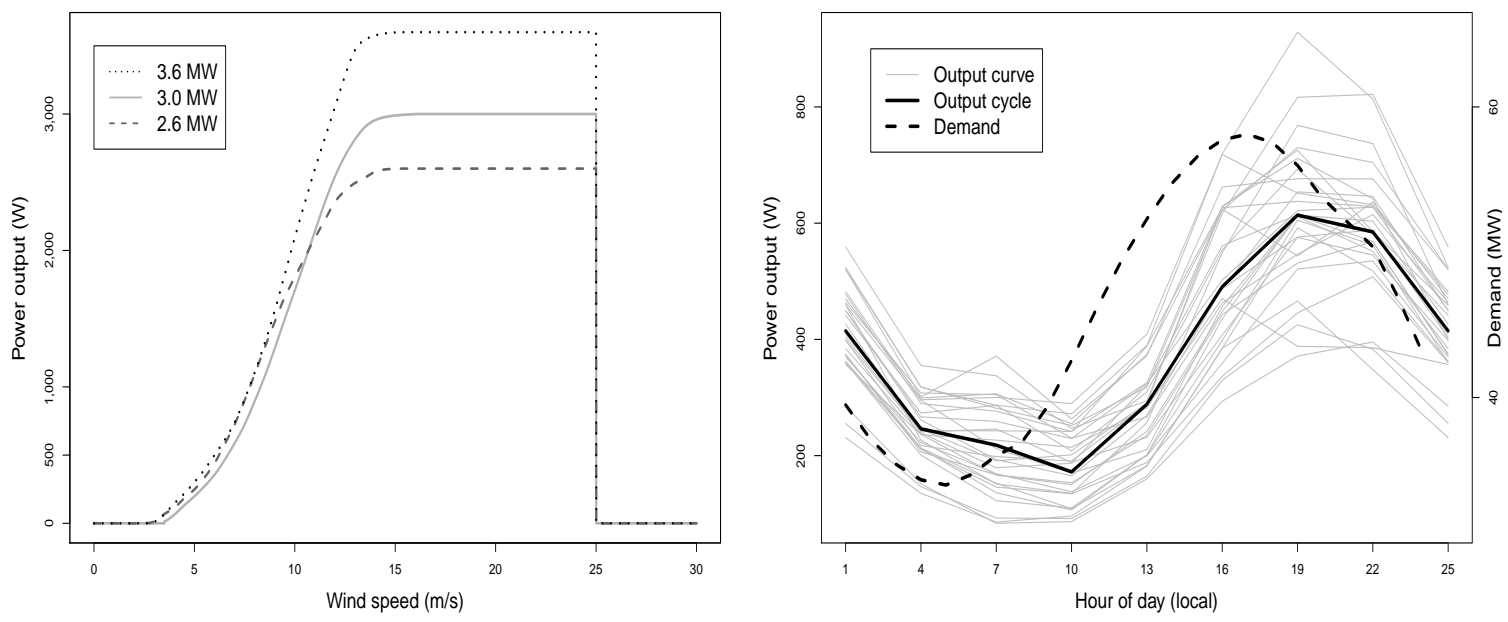

Figure 1: Power output functions. Left: Power transformation curves for the Siemens SWT 3.6 MW 120, Vesta V90 3.0 MW, and Vesta V100 2.6 MW wind turbines. Right: Twentynine diurnal power curves plotted with the estimated diurnal cycle for the Texas site using the 3.0 MW turbine power transformation. The hour of the day is plotted along the horizontal axis, and the power output is plotted along the vertical axis. Also overlaid is an example of a diurnal load cycle. 


\section{Summer Results}

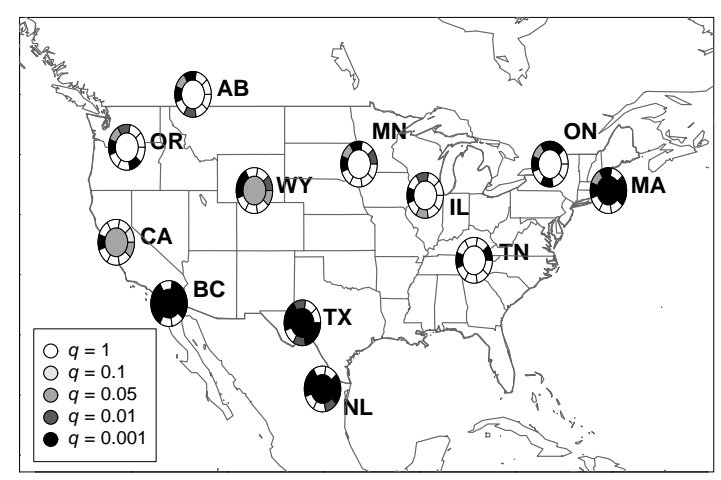

Winter Results

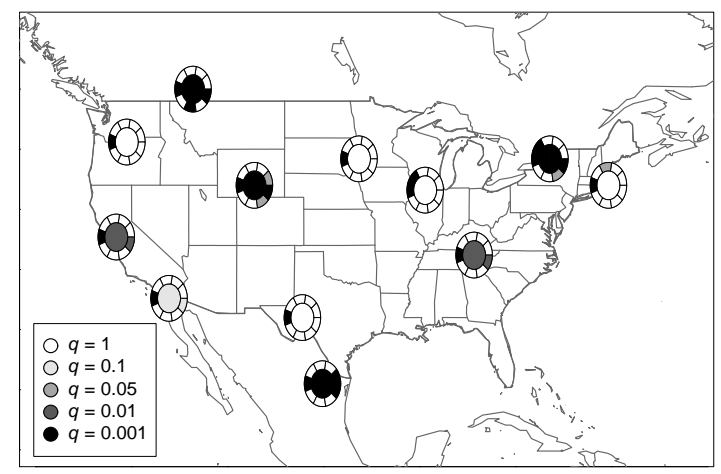

Autumn Results

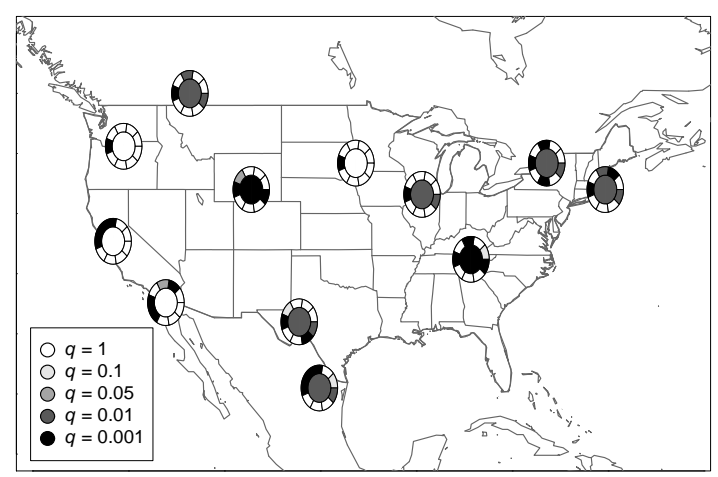

Spring Results

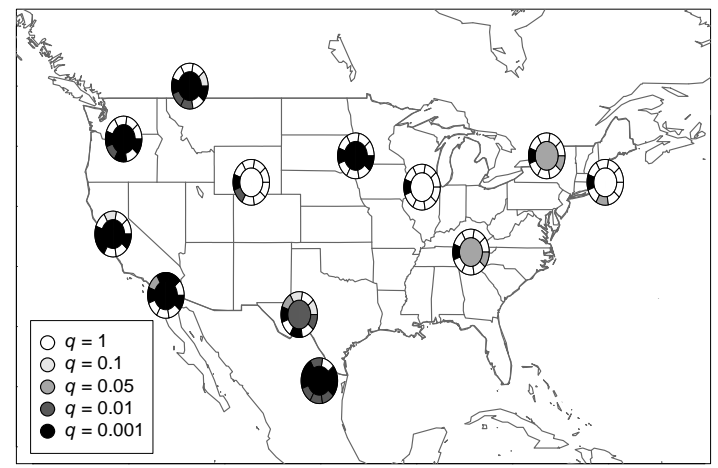

Figure 2: Results of the semi-parametric test for each location and season. The central disc is shaded proportionally to the lowest false discovery rate, $q$, at which the averaged-catalog test is significant. The outer ring is segmented so that each region reports the test result of an individual RCM catalog and is shaded in the same manner as the discs. Segments, beginning at the " 1 o'clock" position and continuing clockwise, correspond to the catalogs in the order that they are listed in Section 3.1. Maps created with R software (R Core Team 2012). 

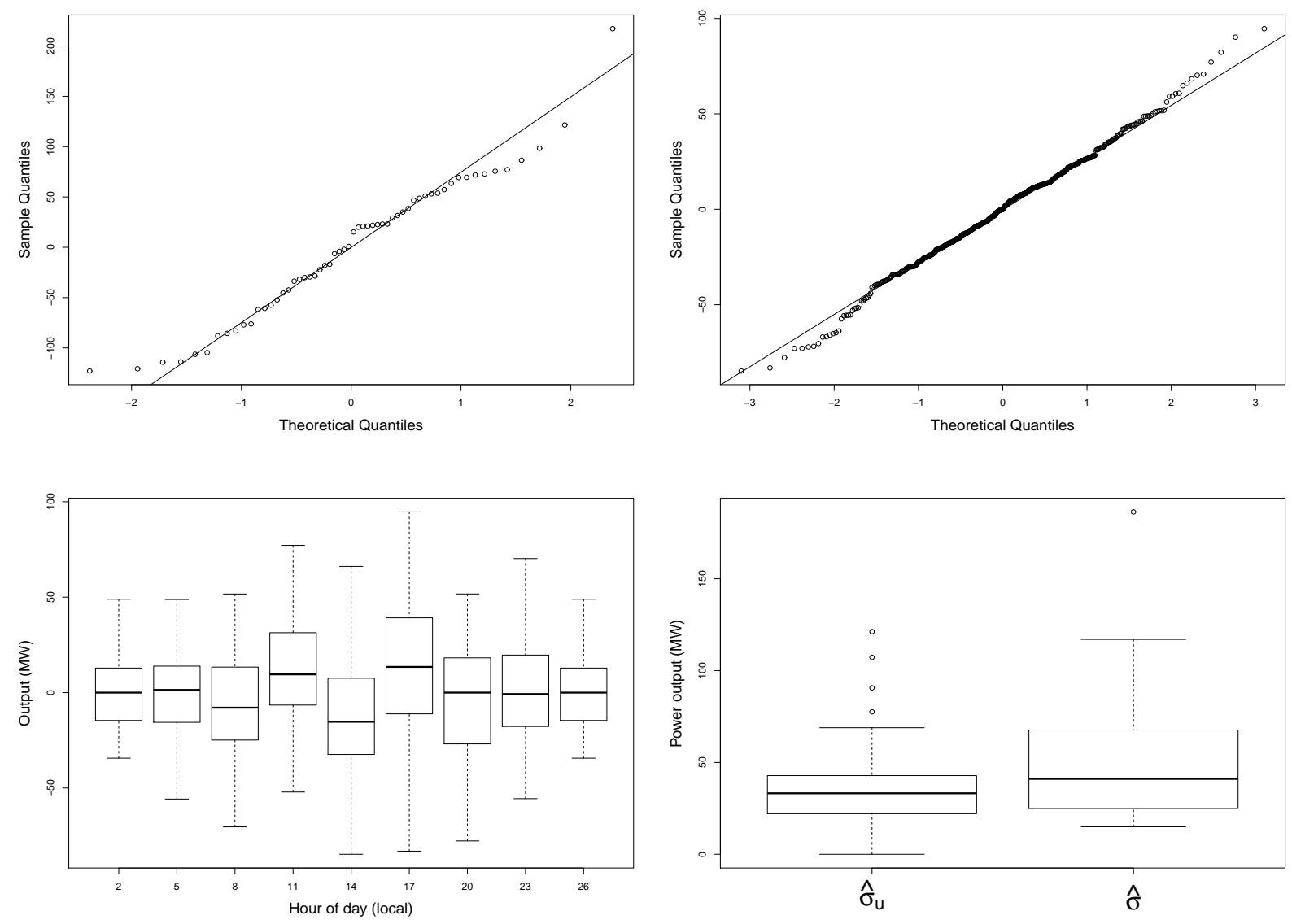

Figure 3: A sampling of assessments of testing assumptions and model fit. Top row: Normal probability plots of $\hat{\mathbf{u}}$ (left) and $\hat{\mathbf{e}}$ (right) for MA (summer). Bottom row: Distributions of $\hat{\mathbf{e}}$ at each time of day for MA (summer) for assessing homoscedasticity (left) and distributions of $\hat{\sigma}_{u}$ and $\hat{\sigma}$ for all season-locations (right). 

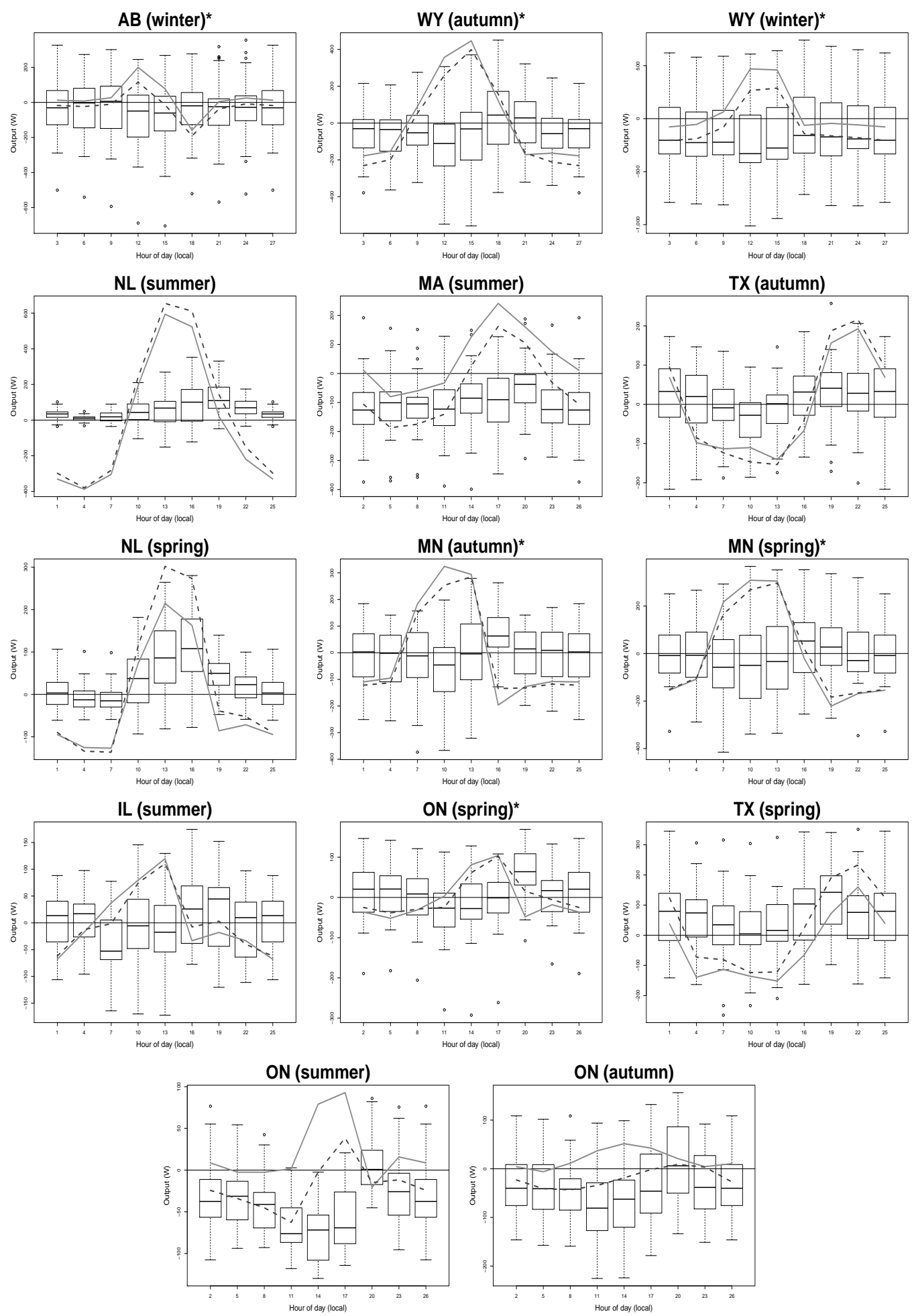

Figure 4: Mean differenced boxplots for the 14 averaged-catalog season-location tests whose result is significant at $q=0.001$ and whose relevant assumptions are strongly or moderately satisfied. The top two rows consist of season-location sites where the mean diurnal cycle shifts vertically. The next two rows of season 3 18cations indicate a change in amplitude in the mean diurnal cycle. The final row consists of more complicated changes. Asterisks indicate season-location tests for which the assumptions are strongly validated. Overlaid curves are the averaged diurnal historical cycle (solid line) and future cycle (dashed line). 